\title{
ON THE CPA IN A MUFFIN-TIN MODEL POTENTIAL THEORY OF RANDOM SUBSTITUTIONAL ALLOYS
}

\author{
B. L. GYORFFY and G. M. STOCKS
}

HH Wills Physics Laboratory, Bristol University, U. K.

\begin{abstract}
Résumé. - Nous considérons un alliage binaire aléatoire. Les équations fondamentales satisfaites par l'amplitude effective de diffusion $t_{\mathrm{L}}^{\mathrm{C}}(\varepsilon)$ dans l'approximation CPA se simplifient pour des puits de potentiel muffin-tin sans recouvrement, et nous pourrons dériver une nouvelle formule pour la densité d'états, $n(\varepsilon)$, en termes de $t_{\mathrm{L}}^{\mathrm{C}}(\varepsilon)$. Nous montrons qu'à la limite des faibles densités, $n(\varepsilon)$ est donnée par la moyenne de la somme de Friedel

$$
\frac{1}{\pi} \Sigma(2 l+1)\left\{c \delta_{\mathrm{e}}^{\prime A}+(1-c) \delta_{\mathrm{e}}^{\prime \mathrm{B}}\right\}
$$

où $\delta_{\mathrm{e}}^{\prime \mathbf{A}}$ et $\delta_{\mathrm{e}}^{\prime \mathbf{B}}$ sont les dérivés (en énergies) des déphasages correspondant aux potentiels $\mathbf{A}$ et $\mathbf{B}$ respectivement. Nous proposons donc une représentation particulièrement suggestive de $t_{\mathrm{L}}^{\mathrm{C}}$ selon des diagrammes d'Argand et démontrons l'utilité de cette représentation en calculant $n(\varepsilon)$ pour un $t_{\mathrm{L}}^{\mathrm{d}}(\varepsilon)$ approximatif, adéquat pour un alliage $\mathrm{Ni}-\mathrm{Cu}$. Compte tenu de la discussion précédente, nous introduisons une nouvelle approximation pour résoudre les équations fondamentales de la CPA pour $t_{x}^{\mathrm{C}}$. Cette méthode est pratique et peut nous permettre de traiter les bandes-d quelles que soient (i) leurs largeurs (ii) la séparation des énergies résonnantes et (iii) l'hybridisation s-d.
\end{abstract}

Abstract. - We consider a random binary alloy. The fundamental equations satisfied by the effective scattering amplitude $t_{\mathrm{L}}^{\mathrm{C}}(\varepsilon)$ in the CPA for non-overlapping muffin-tin potential wells is simplified and a new formula for the density of states, $n(\varepsilon)$, in terms of $t_{\mathrm{L}}^{\mathrm{C}}(\varepsilon)$, is derived. It is shown that in the low density limit $n(\varepsilon)$ is given by the averaged Friedel sum, where $\delta_{l}^{\prime A}$ and $\delta_{l}^{\prime \mathbf{B}}$ are the energy derivatives of the $l$-th phase-shifts corresponding to the $\mathbf{A}$ and $\mathbf{B}$ potentials respectively. We then propose a particularly suggestive representation for $t_{\mathrm{L}}^{\mathrm{C}}$ in terms of Argand plots and demonstrate the utility of this representation by calculating $n(\varepsilon)$ for an approximate $t_{\mathrm{L}}^{\mathrm{C}}(\varepsilon)$ appropriate to a $\mathrm{Ni}-\mathrm{Cu}$ alloy. Based on the insight gained in the foregoing discussion we introduce a new approximation for solving the fundamental equations of CPA for $t_{\mathrm{L}}^{\mathrm{C}}$ and argue that this scheme is feasible and will allow us to treat d-bands with arbitrary band widths, arbitrary separation of resonant energies and any amounts of s-d hybridization.

1. Introduction. - By now, there are a vast number of calculations in the literature [1] using the CPA in the context of simplified, usually tight binding, model Hamiltonians. Together, these appear to indicate that the basic principles involved are sufficiently sound to make the CPA prescription, in its model independent form, a reasonable starting point for more elaborate treatments of the electronic structure in random substitutional, metallic, alloys. Our purpose here is to discuss the feasibility and the desirability of carrying out the CPA program for a crystal potential which while varying randomly from site to site is of the non-over-lapping muffin-tin form. We have in mind potentials constructed according to the Mattheiss [2] prescription as is customary in the band theory of ordered metallic alloys [3]. We shall discuss the problem in the language of multiple scattering theory and, therefore, the theory will be analogous to and on the scale of a KKR [4] band structure calculation.

The formal solution of the problem has been given by Soven [5], Shiba [6] and Gyorffy [7]. They showed that the CPA for a random binary alloy whose crystal potential is represented by a set of non-overlapping, spherically symmetric, muffin-tin potential wells entails finding an effective single site scattering amplitude $t_{\mathrm{C}, \mathrm{L}}(\varepsilon)$, where $\mathrm{L}$ stands for both angular momentum quantum numbers $l$ and $m$. The condition which determines $t_{\mathrm{C}, \mathrm{L}}(\varepsilon)$ is that the appropriate, ordered arrangement of scattering centers each scattering electrons according to $t_{\mathrm{C}, \mathrm{L}}$, would represent the average behaviour of the random lattice. This led to an implicit equation for $t_{C, L}$ involving the angular momentum components, $t_{\mathrm{A}, \mathrm{L}}(\varepsilon)$ and $t_{\mathrm{B}, \mathrm{L}}(\varepsilon)$ of the « on the energy shall » $t$-matrices which describe the scattering properties of an $A$ well and $B$ well, respectively, and the structure constants $G_{\mathrm{LL}}^{+}(\mathbf{q}, \varepsilon)$ characteristic of the crystal lattice. It is rather straight forward to show, starting with the equation for $t_{\mathrm{C}, \mathrm{L}}$ given by Shiba [5] and Gyorffy [6] that

$$
\begin{aligned}
t_{\mathrm{C}, \mathrm{L}}^{-1}=c t_{\mathrm{A}, \mathrm{L}}^{-1}+(1-c) t_{\mathrm{B}, \mathrm{L}}^{-1} & +\left(t_{\mathrm{C}, \mathrm{L}}^{-1}-t_{\mathrm{A}, \mathrm{L}}^{-1}\right) \times \\
& \times J_{\mathrm{LL}^{\prime}, 00}^{\mathrm{C}, 0)\left(t_{\mathrm{C}, \mathrm{L}}^{-1}-t_{\mathrm{B}, \mathrm{L}}^{-1}\right)}
\end{aligned}
$$

where

$$
J_{\mathrm{LL}^{\prime}}^{\mathrm{C}, 00}(\varepsilon)=\frac{1}{\tau} \int \mathrm{d}^{3} q\left[\frac{1}{t_{\mathrm{C}}^{-1}-G^{+}(\mathbf{q}, \varepsilon)}\right]_{\mathrm{LL}^{\prime}}
$$


the integral over the Brillouin zone of the inverse KKR matrix.

Perhaps it is helpful to note that eq. (1) is in one to one correspondence with the equation for the CPA single site self energy $\Sigma_{\mu}(\varepsilon)$ in the usual tight binding formulation of this problem. In place of the index $\mu$ which, in the tight binding language, specifies a particular atomic orbital, in eq. (1) we have the angular momenta $\mathrm{L}$. The familiar $\varepsilon-\varepsilon_{\mu}^{\mathrm{A}}, \varepsilon-\varepsilon_{\mu}^{\mathrm{B}}$ and $\varepsilon-\Sigma_{\mu}(\varepsilon)$ are equivalent to $t_{\mathrm{A}, \mathrm{L}}^{-1}, t_{\mathrm{B}, \mathrm{L}}^{-1}$ and $t_{\mathrm{C}, \mathrm{L}}^{-1}$, respectively, and $J_{\mathrm{LL}^{\prime}}^{\mathrm{C}, 00}(\varepsilon)$ plays the role of the site diagonal part of the Green function $G_{0 \mu, 0 \mu^{\prime}}^{+}(\varepsilon)$. This correspondence is, of course, not coincidental. It is the consequence of the pseudo-tight binding form of the multiple scattering equations on the energy shell [8,9].

Thus, our fundamental CPA equation is eq. (1). However, before beginning to discuss ways of solving it we must settle one furher issue. Namely, we must say what is to be done with $t_{\mathrm{C}, \mathrm{L}}(\varepsilon)$ once it has been obtained. Unfortunately, this turns out to be a nontrivial problem. Without attempting to answer it in full generality we shall discuss how to calculate the density of states from $t_{\mathrm{C}, \mathrm{L}}(\varepsilon)$.

2. On calculating the averaged density of states. It was suggested by Gyorffy [7] that once $t_{\mathrm{C}, \mathrm{L}}(\varepsilon)$ has been determined the integrated density of states may be calculated from the formula [9]

$$
\begin{aligned}
N(\varepsilon)=N^{\circ}(\varepsilon)=\frac{1}{\pi} & \operatorname{Im} \int \mathrm{d}^{3} q \times \\
& \times \ln \left\|t_{\mathrm{C}, \mathrm{L}}^{-1} \delta_{\mathrm{LL}^{\prime}}-G_{\mathrm{LL}}^{+}(\mathbf{q}, \varepsilon)\right\|
\end{aligned}
$$

where $N^{0}(\varepsilon)$ is the free particle integrated density of states and the integral is over the Brillouin zone. This is an appealing result since for a real, energy independent muffin-tin well $t_{\mathrm{L}}^{-1}(\varepsilon)=-\sqrt{\varepsilon}\left(\cot \delta_{l}-i\right)$ where $\delta_{l}$ 's are the usual scattering phase shifts, and therefore, at least for a cubic lattice, the determinant is real. Consequently, the operation of taking the imaginary part and integrating over the Brillouin zone merely counts the number of zeros

$$
\left\|t_{\mathrm{L}}^{-1} \delta_{\mathrm{LL}^{\prime}}-G_{\mathrm{LL}}^{+}(, \mathbf{q} \varepsilon)\right\|
$$

has under a constant energy surface. But this is just the constant energy search KKR calculation of Faulkner, Davis and Joy [10]. Thus, the use of eq. (3) for $t_{\mathrm{C}, \mathrm{L}}^{-1}$ would appear to be its natural generalization. Unfortunately, it is not correct. To see this, note that in the low density limit (to obtain it one merely has to neglect $G_{L^{\prime}}^{+}(\mathbf{q}, \varepsilon)$ in eq. (3)) where $\frac{N}{\Omega} \rightarrow 0$. Eq. (3) gives

$$
N(\varepsilon)=N^{0}(\varepsilon)-\frac{N}{\Omega} \operatorname{Im} \sum_{\mathbf{L}} \ln t_{\mathrm{C}, \mathbf{L}}^{-1}
$$

the Friedel sum for the effective scatterers. Solving eq. (1) in the same approximation gives

$$
t_{\mathrm{CL}}=c t_{\mathbf{L}}^{\mathbf{A}}+(1-c) t_{\mathbf{L}}^{\mathbf{B}}
$$

and hence we have

$N(\varepsilon)=N^{0}(\varepsilon)+\frac{N}{\Omega} \operatorname{Im} \sum_{\mathrm{L}} \ln \left[c t_{\mathrm{A}, \mathrm{L}}+(1-c) t_{\mathrm{B}, \mathrm{L}}\right]$

which is manifestly not equal to the exact low density result :

$$
N(\varepsilon)=N^{0}(\varepsilon)+\frac{N}{\Omega} \operatorname{Im} \sum_{\mathrm{L}}\left[c \ln t_{\mathrm{A}, \mathrm{L}}+(1-c) \ln t_{\mathrm{B}, \mathrm{L}}\right]
$$

However, it can be shown in a representation independent way that the CPA is exact in the low density limit. It is a bit more complicated but it can also be shown that eq. (3) leads to the wrong answer in the low concentration limit, $c \ll 1$, where CPA is also known to be exact.

The difficulty arises because eq. (3) is equal to the integrated trace of the Green's function only for energy independent potentials and the potential corresponding to $t_{\mathrm{C}, \mathrm{L}}$ is not energy independent. Though the CPA prescription tells us that the trace of the coherent potential Green's function is the average density of states regardless whether the coherent potential is energy dependent or not we have no way of calculating that trace in terms of $t_{\mathrm{C}, \mathrm{L}}(\varepsilon)$. In general it also depends on the « half off the energy shell $\gg$ components of the coherent potential $t$ matrix, $t_{C, L}(\sqrt{\varepsilon}, k ; \varepsilon)$, which is not given by eq. (1).

A way of getting around this problem will suggest itself if we go back to an exact formula for the differential density of states corresponding to an arbitrary configuration. It follows from the work of Lloyd [11] and Gyorffy and Stott [12] that for spherically symmetric, nonoverlapping potentials on a lattice the integrated density of states is given by

$$
N(\varepsilon)=N^{0}(\varepsilon)-\frac{1}{\pi} \operatorname{Im} \ln \left\|t_{\mathrm{i}, \mathrm{L}}^{-1} \delta_{\mathrm{LL}} \delta_{\mathrm{i}, \mathrm{j}}-G_{\mathrm{LL}}^{+}\left(\mathbf{R}_{\mathrm{i}}-\mathbf{R}_{\mathrm{j}}\right)\right\|
$$

where $t_{\mathrm{i}, \mathrm{L}}(\varepsilon)$ 's are the $t_{\mathrm{L}}(\varepsilon)$ for the potential on the site $\mathrm{i}$, $G_{\mathbf{L} \mathbf{L}^{\prime}}^{+}\left(\mathbf{R}_{\mathbf{i}}-\mathbf{R}_{\mathbf{j}}\right)$ is the structure constant for the lattice in configuration space and the determinant is to be taken with respect to the site indices $i$ and $j$ as well as angular momenta $\mathrm{L}$ and $\mathrm{L}^{\prime}$. In our case $t_{\mathrm{iL}}$ 's are $t_{\mathrm{A}, \mathrm{L}}(\varepsilon)$ and $t_{\mathrm{B}, \mathrm{L}}(\varepsilon)$ in a random fashion. Note that the potentials $v^{\mathrm{A}}$ and $v^{\mathrm{B}}$ which give rise to $t_{\mathrm{A}, \mathrm{L}}(\varepsilon)$ and $t_{\mathrm{B}, \mathrm{L}}(\varepsilon)$ respectively, are energy independent so eq. (5) is the integrated trace of the Green's function. Let us now consider the configurational average of this trace :

$$
\begin{aligned}
& \bar{n}(\varepsilon) \equiv\left\langle\frac{\mathrm{d} N(\varepsilon)}{\mathrm{d} \varepsilon}\right\rangle
\end{aligned}
$$

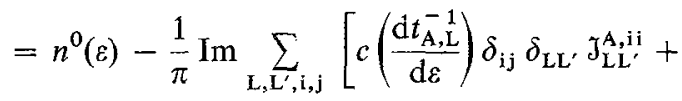

$$
\begin{aligned}
& +(1-c)\left(\frac{\mathrm{d} t_{\mathrm{B}, \mathrm{L}}^{-1}}{\mathrm{~d} \varepsilon}\right) \delta_{\mathrm{ij}} \delta_{\mathbf{L L}^{\prime}} \mathfrak{j}_{\mathbf{L} \mathbf{L}^{\prime}}^{\mathrm{B}, \mathrm{ii}} \\
& \left.-\left(\frac{\mathrm{d} G_{L L^{\prime}}\left(\mathbf{R}_{\mathbf{i}}-\mathbf{R}_{\mathbf{j}}\right)}{\mathrm{d} \varepsilon}\right)\left(1-\delta_{\mathrm{ij}}\right) \overline{\mathfrak{J}}_{\mathbf{L L}^{\prime} \mathbf{j}}\right]
\end{aligned}
$$


where we took the energy derivative of eq. (5) first and performed the configurational average afterwards. Consequently, $J_{\mathrm{LL}^{\prime}}^{\mathrm{A}, \mathrm{ii}}$ is the site diagonal component of the inverse of the generalized KKR matrix

$$
\mathfrak{J}_{\mathrm{LL}^{\prime}}^{\mathrm{ij}}=\left[\frac{1}{t^{-1}-G^{+}}\right]_{\mathrm{LL}}
$$

averaged over all those members of the configuration ensemble which have an $\mathrm{A}$ atom at the site $\mathrm{i}$. $\mathfrak{J}_{\mathbf{L L}}^{\mathrm{B}, \mathrm{ij}}$ is, of course, the same for a B site, and $\bar{J}_{L^{\prime}}^{i j}$ is the fully averaged site off-diagonal component of $\mathcal{J}_{\mathrm{LL}}^{\mathrm{ij}}$.

If we now take eq. (6) as the starting point for a theory of the averaged density of states $\bar{n}(\varepsilon)$ an approximation scheme must give prescriptions for calculating $\mathfrak{J}_{\mathrm{LL}^{\prime}}^{\mathrm{A}, \mathrm{ii}}, \mathfrak{J}_{\mathrm{LL}^{\prime}}^{\mathrm{B}, \mathrm{ii}}, \overline{\mathfrak{J}}_{\mathrm{LL}^{\prime}}^{\mathrm{ij}}$. It is relatively easy to see that in CPA we must take

$$
\begin{aligned}
& {\left[\overline{\mathfrak{J}}^{-1}\right]_{\mathrm{iLjL}}=\hat{t}_{\mathrm{C}, \mathrm{L}}^{-1} \delta_{\mathrm{LL}^{\prime}} \delta_{\mathrm{ij}}-G_{\mathrm{LL}^{\prime}}^{+}\left(\mathbf{R}_{\mathrm{i}}-\mathbf{R}_{\mathrm{j}}\right)} \\
& {\left[\left(\mathcal{J}^{\mathrm{B}}\right)^{-1}\right]_{\mathrm{iL}, \mathrm{jL}}=\left[t_{\mathrm{C}, \mathrm{L}}^{-1} \delta_{\mathrm{ij}}+\left(t_{\mathrm{B}, \mathrm{L}}^{-1}-t_{\mathrm{C}, \mathrm{L}}^{-1}\right) \delta_{\mathrm{i}, 0} \delta_{\mathrm{j}, 0}\right] \delta_{\mathbf{L L}^{\prime}}-} \\
& -G_{\mathrm{LL}}^{+}\left(\mathbf{R}_{\mathrm{i}}-\mathbf{R}_{\mathrm{j}}\right) \\
& {\left[\left(J^{A}\right)^{-1}\right]_{i \mathbf{L}, j \mathbf{L}^{\prime}}=\left[t_{\mathrm{C}, \mathbf{L}}^{-1} \delta_{\mathbf{i j}}+\left(t_{\mathrm{A}, \mathrm{L}}^{-1}-t_{\mathrm{C}, \mathrm{L}}^{-1}\right) \delta_{\mathrm{i}, 0} \delta_{\mathrm{j}, 0}\right] \delta_{\mathbf{L L}^{\prime}}-} \\
& -G_{L L^{\prime}}^{+}\left(\overline{\mathbf{R}}_{\mathbf{i}}-\mathbf{R}_{\mathrm{j}}\right) \text {. }
\end{aligned}
$$

That is to say $\tilde{J}_{\mathrm{LL}^{\prime}}^{\mathrm{ij}}$ is $\mathcal{J}_{\mathrm{LL}}^{\mathrm{ij}}$. for a coherent potential lattice with the scattering on every site described by $t_{\mathrm{C}, \mathbf{L}}$ and $\mathfrak{J}_{\mathbf{L L}^{\prime}}^{\mathrm{A}, 00}, \mathfrak{J}_{\mathbf{L L}^{\prime}}^{\mathrm{B}, 00}$ are the matrices $\mathfrak{J}_{\mathbf{L L}}^{00}$ for an $\mathrm{A}$ or $\mathrm{B}$ impurity, respectively, in the coherent potential lattice at $\mathbf{R}_{\mathbf{0}}$.

With these prescriptions for $\bar{\jmath}_{\mathrm{LL}^{\prime}}^{\mathrm{i}, j}, \mathfrak{J}_{\mathbf{L}^{\prime}}^{\mathrm{A}, 00}$ and $\jmath_{\mathrm{LL}^{\prime}}^{\mathrm{B}, 00}$ it is straight forward but lengthy to show that

$$
\begin{aligned}
\bar{N}(\varepsilon) & = \\
=N^{0}(\varepsilon) & -\frac{1}{\pi} \operatorname{Im} \frac{1}{\tau} \int \mathrm{d}^{3} q \ln \left\|t_{\mathrm{C}, \mathrm{L}}^{-1} \delta_{\mathrm{LL}^{\prime}}-G_{\mathrm{LL}}^{+}(\mathbf{q}, \varepsilon)\right\| \\
& -\frac{c}{\pi} \operatorname{Im} \ln \left\|\frac{t_{\mathrm{B}, \mathrm{L}}^{-1}-<t_{\mathrm{L}}^{-1}>}{t_{\mathrm{B}, \mathrm{L}}^{-1}-t_{\mathrm{C}, \mathrm{L}}^{-1}}\right\| \\
& -\frac{1-c}{\pi} \operatorname{Im} \ln \left\|\frac{t_{\mathrm{A}, \mathrm{L}}^{-1}-<t_{\mathrm{L}}^{-1}>}{t_{\mathrm{A}, \mathrm{L}}^{-1}-t_{\mathrm{C}, \mathrm{L}}^{-1}}\right\|
\end{aligned}
$$

where

$$
\bar{N}(\varepsilon)=\int_{0}^{\varepsilon} \mathrm{d} \varepsilon \bar{n}(\varepsilon)
$$

and

$$
\left\langle t_{\mathrm{L}}^{-1}\right\rangle=c t_{\mathrm{A}, \mathrm{L}}^{-1}+(1-c) t_{\mathrm{B}, \mathrm{L}}^{-1} .
$$

The details of the mathematics leading to eq. (8) will be published elsewhere [13]. Here we merely want to note that the simple, single site, form of the correction to eq. (3) is the result of repeated use of the CPA condition : $c \mathcal{J}_{\mathrm{LL}^{\prime}}^{\mathrm{A}, 00}+(1-c) \mathfrak{J}_{\mathrm{L}^{\prime}}^{\mathrm{B}, 0,}=\mathcal{J}_{\mathrm{LL}^{\prime}}^{\mathrm{C}, 0,}$ from which eq. (1) also follows. Hence eq. (8) is not a general formula : its validity depends on the use of a self consistently determined scattering matrix $t_{\mathrm{C}, \mathrm{L}}(\varepsilon)$.
Thus doing the CPA for nonoverlapping muffin-tin wells consists of solving eq. (1) for $t_{C, L}$ and using eq. (8) for calculating the averaged integrated density of states.

Neglecting $G_{\mathrm{I} X}^{+}(\mathbf{q}, \varepsilon)$ in eq. (8) it is straight forward to show that it gives, in this low density, i. e. $N / \Omega \rightarrow 0$, limit the exact result given eq. (5). Also, as will be shown elsewhere [13], in the small concentration limit, namely to the order $c$ (eq. (8)) gives

$$
\bar{N}(\varepsilon)=N^{A}(\varepsilon)-\frac{c}{\pi} \operatorname{Im} \ln \left\|\delta_{\mathrm{LL}^{\prime}}+\left(t_{\mathrm{B}, \mathrm{L}}^{-1}-t_{\mathrm{A}, \mathrm{L}}^{-1}\right) \mathcal{J}_{\mathrm{LL}}^{00}\right\|
$$

where $N^{\mathrm{A}}(\varepsilon)$ is the integrated density of states for the pure. A host lattice and $\int_{\mathrm{LL}^{\prime}}^{00}$ is given by eq. (2) with the host lattice $t_{\mathrm{A}, \mathrm{L}}$ replacing $t_{\mathrm{C}, \mathrm{L}}$. It is reassuring to note that eq. (9) is exact.

Having defined explicitly what the CPA program means for our model Hamiltonian we shall, in the next section, discuss same approximate ways of carrying it out.

3. Inelastic scatterers and randomness. - For a spherically symmetric real potential

$$
t_{\mathrm{L}}(\varepsilon)=i_{l}(\varepsilon)=-\frac{1}{\sqrt{\varepsilon}} \sin \delta_{l} \mathrm{e}^{\mathrm{i} \delta_{l}}
$$

where $\delta_{l}$ 's are the scattering phase shifts, and they are real. The coherent potential which corresponds to $t_{C, L}$ will be complex hence we expect the phase shifts to be complex as well. That is to say $t_{\mathrm{C}, \mathrm{L}}$ describes an inelastic scattering process. Under these circumstances it is more convenient to talk in terms of scattering amplitudes $f_{l}(\varepsilon)$ which are related to $t_{l}(\varepsilon)$ by

$$
f_{l}(\varepsilon)=-\sqrt{\varepsilon} t_{l}(\varepsilon)
$$

For a real potential, i. e., elastic scatterer, it follows from eq. (10) that

$$
f_{l}(\varepsilon)=\frac{1}{2 \mathrm{i}}\left(\mathrm{e}^{2 \mathrm{i} \delta_{1}}-1\right) .
$$

A scattering amplitude which corresponds to an inelastic scattering processes $f_{\mathrm{C}, l}$ is customarily parametrized $[14]$ as

$$
f_{\mathrm{C}, l}(\varepsilon)=\frac{1}{2 \mathrm{i}}\left(\alpha_{l} \mathrm{e}^{2 \mathrm{i} \eta_{l}}-1\right)
$$

where $\alpha_{l}$ and $\eta_{l}$ are real numbers and, of course are, functions of energy $\varepsilon$. The function $\eta_{l}(\varepsilon)$ plays the same role of the phase shift in an elastic process, i. e., it is a measure of the strength of the interaction while $\alpha_{l}(\varepsilon)$ measures the degree of inelasticity. That is to say the degree to which the scattering center acts as a sink for the particles.

A useful graphical representation of an inelastic scattering amplitude is an Argand plot. This is obtained by plotting $\operatorname{Im} f_{l}$ against Rel $f_{l}$. An example is shown in figure 1 . The dotted line represents the uni- 


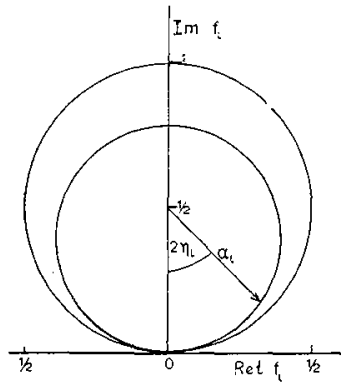

(a)

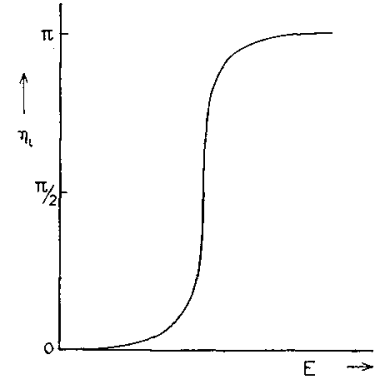

(b)
FIG. 1. - a) Argand plot for the scattering amplitude $f_{l}(\varepsilon)$ which describes an inelastic scatterer.

b) The phase shift $\eta_{l}(\varepsilon)$ corresponding to $f_{l}(\varepsilon)$ in figure 1a as a function of energy.

tarity circle. It is a universal curve for a process with $\alpha=1$ and represents it the equation

$$
\left(\operatorname{Rel} f_{l}\right)^{2}+\left(\operatorname{Im} f_{l}-\frac{1}{2}\right)^{2}=\frac{1}{4}
$$

which follows from eq. (11). The full line is described by the equation

$$
\left(\operatorname{Rel} f_{l}\right)^{2}+\left(\operatorname{Im} f_{l}-\frac{1}{2}\right)^{2}=\frac{\alpha^{2}}{4}
$$

which is a trivial consequence of eq. (12). Both curve corresponds a resonant phase shift $\eta_{l}(\varepsilon)$ shown in figure $1 b$. Thus we see that the radius of the Argand plot measures the probability that an electron is lost in the scattering process.

Clearly, $t_{\mathrm{C}, \mathrm{L}}$ obtained from solving eq. (1) will correspond to an inelastic scattering amplitude $f_{\mathrm{C}, l}$. The inelasticity, $\alpha_{l}$, is due to the randomness in the problem. Thus, we are describing average behaviour of our random alloy by considering an electron propagating according to the free particle propagators $G_{\mathbf{L L}}^{+},\left(\mathbf{R}_{\mathbf{i}}-\mathbf{R}_{\mathbf{i}}\right)$ between an ordered arrangement of inelastic scattering centers. This is a description of the randomness which is equivalent to considering an electron propagating with a complex wave vector or being in momentum eigenstate with complex energy. In fact, roughly speaking, the zeros of the determinant $\left\|t_{\mathrm{C}, \mathrm{L}}^{-1} \delta_{\mathrm{LL}}-G_{\mathrm{LL}}^{+}(\mathbf{q}, \varepsilon)\right\|$ may be thought of as a complex band structure [15]. Evidently, the width of the levels will arise from the inelasticity in the individual scattering processes.

While our aim is to carry out the full CPA program, at this stage of the theory it is clearly desirable to find some fairly realistic but computationally more tractible approximation to it. Consider an alloy of two d-band metals and assume, for the sake of simplicity, that their $\mathrm{s}$ and $\mathrm{p}$ phase shifts are the same. Allow, however, the two d resonances to have different shapes and be shifted with respect to each other. Then, we may expect that in the energy range around the two resonance the CPA scattering amplitude will be highly inelastic. This suggests a way of approximating eq. (1). Note that in the direct lattice representation $J_{L^{\mathrm{C}} \mathrm{L}^{\prime}}^{\mathrm{C}, 00}$ is given by an expansion

$$
\begin{aligned}
\mathfrak{J}_{\mathrm{LL}^{\prime}}^{\mathrm{C}, 00}=t_{\mathrm{C}, \mathrm{L}} \delta_{\mathrm{LL}^{\prime}}+ & \sum_{\mathrm{L}^{\prime \prime}, \mathrm{i} \neq 0} t_{\mathrm{C}, \mathrm{L}} G_{\mathrm{LL}^{\prime \prime}}^{+}\left(\mathbf{R}_{0}-\mathbf{R}_{\mathrm{i}}\right) \times \\
& \times t_{\mathrm{C}, \mathrm{L}^{\prime \prime}} G_{\mathrm{L}^{\prime \prime} \mathrm{L}^{\prime}}^{+}\left(\boldsymbol{R}_{\mathrm{i}}-\mathbf{R}_{0}\right) t_{\mathrm{C}, \mathrm{L}^{\prime}}+\cdots
\end{aligned}
$$

which is the sum over all scattering paths which start from $\mathbf{R}_{0}$ and return to $\mathbf{R}_{0}$. Note also that in each scattering process the particle wave will lose intensity due to the inelastic nature of this process. Thus one might expect that the self consistency condition on the site $\mathbf{R}_{0}$, namely eq. (1) will give approximately the right $t_{\mathrm{C}, \mathrm{L}}(\varepsilon)$ even if we did not use the exact $\Im_{\mathrm{LL}^{\prime}}^{\mathrm{C}, 00}$ but included only those paths in eq. (13) which involve only a few scattering events. The most extreme of such approximations would be to take $\mathfrak{J}_{\mathbf{L}^{\prime}}^{\mathrm{C}, 0 .}=t_{\mathrm{C}, \mathrm{L}} \delta_{\mathrm{LL}^{\prime}}$ which we call, for obvious reasons, the local CPA. Substituting $J_{\mathrm{LL}^{\prime}}^{\mathrm{C}, 00}=t_{\mathrm{C}, \mathrm{L}} \delta_{\mathrm{LL}^{\prime}}$ into eq. (1) we can solve for $t_{C, L}$. The solution is

$$
t_{\mathrm{C}, \mathrm{L}}=c t_{\mathrm{A}, \mathrm{L}}+(1-c) t_{\mathrm{B}, \mathrm{L}}
$$

that is to say $t_{C, L}$ is just the average $t$-matrix. We must stress, however, that the local CPA is not the same as the average $t$-matrix approximation (ATA) [16] because we propose to evaluate $N(\varepsilon)$ using eq. (8) which does not follow in ATA. In the local CPA eq. (14) is an approximation to $t_{\mathrm{C}, \mathrm{L}}$ dependent on the fact that $t_{C, L}$ represents strongly inelastic scatterers. Thus, we have a well defined internal criteria by which to judge how good an approximation eq. (14) is. Ways of performing such analysis will be presented elsewhere [13]. Here, in the next section, we merely want to show the results of a few preliminary calculations using this local approximation to the CPA.

4. Density of states for Ni-Cu alloys using the local CPA. - Using the Matthiess [2] prescription we have constructed muffin-tin potentials for a $\mathrm{Ni}$ and $\mathrm{Cu}$ lattice. From the corresponding phaseshifts $\delta_{l}^{\mathrm{Cu}}$ and $\delta_{l}^{\mathrm{Ni}}$ we calculated the averaged scattering amplitude

$$
f_{\mathrm{C}, l}=\bar{f}_{l}=\frac{c}{2 \dot{\mathrm{i}}}\left(\mathrm{e}^{2 \mathrm{i} \delta} \bar{c}_{l}^{\mathrm{u}}-1\right)+\frac{1-c}{2 \mathrm{i}}\left(\mathrm{e}^{2 \mathrm{i} \delta_{l}^{\mathrm{Ni}}}-1\right)
$$

taking the inter scatterer constant potential $V_{\mathrm{MTZ}}$ as the average $c V_{\mathrm{MT}}^{\mathrm{Cu}}+(1-c) V_{\mathrm{MT}}^{\mathrm{Ni}}$ where $V_{\mathrm{MT}}^{\mathrm{Cu}}$ is the muffin-tin zero in the $\mathrm{Cu}$ lattice and $V_{\mathrm{MT}}^{\mathrm{Ni}}$ is the same for the Ni lattice defined in the usual [17] way. The Argand plot for $\overline{f_{l}}$, corresponding to $\delta_{l}^{\mathrm{Cu}}$ and $\delta_{l}^{\mathrm{Ni}}$ given in figure 2, is shown in figure 3 for three different concentrations. It should be clear from the foregoing discussion in Sec. III that these curves should be read as representing a pair of inelastic scattering resonances. For instance, in figure $3 \mathrm{a}$, for $\mathrm{Cu}_{.90} \mathrm{Ni}_{.10}$, we have a fairly elastic resonance, the main loop with radius relatively close to $\frac{1}{2}$, and a severely damped resonance at the high energy side represented by the small loop. This suggests that from $\bar{f}_{l}$ at this concentration we will 


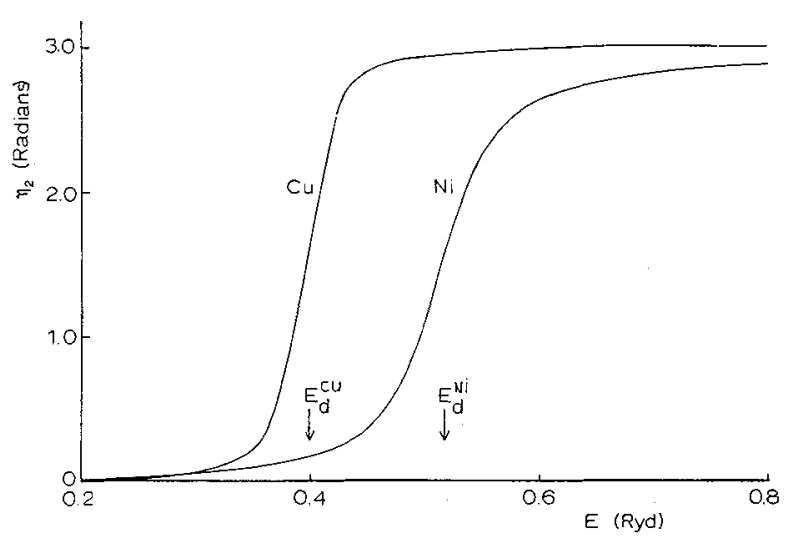

FIG. 2. - The d-phase shifts for the $\mathrm{Cu}$ and Ni muffin-tin potentials used in the calculation.

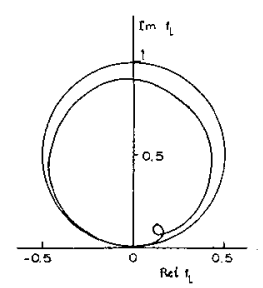

(st

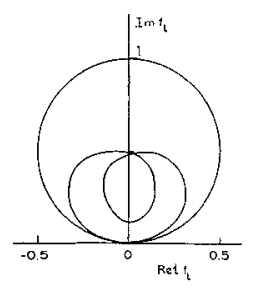

(b)

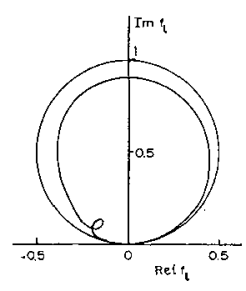

FIG. 3. - Argand plots of the average scattering amplitude $\overline{f_{2}}$ for $\mathrm{Cu} .1 \mathrm{Ni} .{ }_{90}, \mathrm{Cu}_{.50} \mathrm{Ni} .{ }_{50}, \mathrm{Cu} .{ }_{90} \mathrm{Ni}_{11}$ alloys.

mainly get a slightly damped $\mathrm{Cu}$ band structure with severe damping and an extra resonance at the resonance energy of the $\mathrm{Ni}$ potential. This is a picture obviously appealing to the physical intuition and speaks well for the descriptive power of the Argand plot.

In figure 4 we show the results of evaluating eq. (8) with $t_{\mathrm{C}, \mathrm{L}}=-\frac{1}{\sqrt{\varepsilon}} \bar{f}_{l}$ and differentiating the result with respect to $\varepsilon$. With dotted lines we also show the densities of states obtained for these alloys by Stocks, Williams and Faulkner [18] using CPA in the context of a tight binding model Hamiltonian. The agreement between the two calculations is gratifying. This is particularly so since the results of Stocks, Williams and Faulkner are known to be in good accord with a number of experiments on $\mathrm{NiCu}$ alloys [18].

Furthermore, this comparison is perfectly legitimate since the parameters in the model Hamiltonian of Stocks, Williams and Faulkner were obtained from essentially the same $\mathrm{Cu}$ and $\mathrm{Ni}$ phase shifts we have

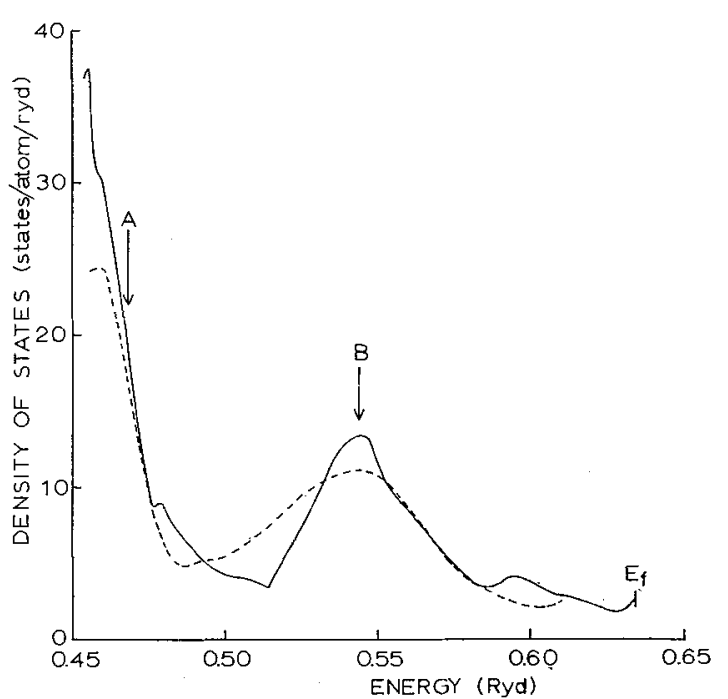

FIG. 4. - The full line is the averaged density of states obtained in the local CPA calculation for $\mathrm{Cu} .90 \mathrm{Ni} 10$. The dotted line is the same as obtained by Stocks et al. in their tight binding model Hamiltonian CPA calculation for $\mathrm{Cu} .87 \mathrm{Ni}_{13}$. Note that the difference to some extent is due to the difference in concentration. Arrow A marks the upper edge of the "copper » d-band and arrow $\mathrm{B}$ indicates the center of the $\mathrm{Ni}$ impurity sub-band.

used in the above calculations and random hopping integrals, differences in phase shifts other than $l=2$ are thought not to play an important role in this alloy system. Consequently, with a bit of caution, one might take the results of our calculations as indicating that to local CPA is a reasonable good approximation to the full CPA.

If confirmed by further calculations this last statement will have a considerable significance. The point is this : to calculate $\bar{f}_{t}$ is trivial, to evaluate the right hand side of eq. (8) is no more difficult or time consuming than a constant energy search KKR calculation. Hence, the local CPA makes a realistic density of states for a random alloy almost as readily accessible as that for a pure metal.

Finally, the method is not limited to alloys whose constituents have similarly shaped bands and closely lying resonances. We hope to present calculations of the density of states in $\mathrm{Cu}-\mathrm{Zn}$ alloys using the present method shortly.

Acknowledgments. - One of us, B. L. G., would like to thank the Oak Ridge National Laboratory for the hospitality during the period this manuscript was prepared. 


\section{References}

[1] SOven, P., Phys. Rev. 156 (1967) 809;

Soven, P., Phys. Rev. 178 (1969) 9936;

VelickÝ, B., KirkPatrick, S. and Ehrenreich, H., Phys. Rev. 175 (1968) 747

Blackman, J. A., Berk, N. F. and Esterling, D. M., Phys. Rev. B 4 (1971) 2412;

Stocks, G. M., Williams, R. W. and Faulkner, J. S. Phys. Rev. B 4 (1971) 4390. J. Phys. F (1973);

Levin, K. and Ehrenreich, H., Phys. Rev. B 3 (1971) 4172 to mention a few.

[2] Mattheiss, L. F., Phys. Rev. 133 A (1964) 1399.

[3] Mattheiss, L. F., Phys. Rev. B 5 (1972) 315.

[4] Korringa, J., Physica 13 (1947) 392 ;

KoHN, W. and Rostoker, Phys. Rev. 94 (1954) 1111

[5] Soven, P., Phys. Rev. B 2 (1970) 4715.

[6] Shiba, H., Progr. Theoret. Phys. (Kyoto) 16 (1971) 77.

[7] Gyorffy, B. L., Phys. Rev. B 5 (1972) 2382.

[8] Heine, Y., Phys. Rev. 153 (1967) 673.

[9] Pettrfore, D. G., J. Phys. C, 5 (1972) 97. Also Gyorfry, B. L. and STOT, M. J. « Band Structure Spectroscopy of Metals and Alloys" (Ed. D. J. Fabian and L. M. Watson) 1973 p. 385.
[10] Faulkner, J. S., Davis, H. L. and Joy, H. W., Phys. Rev. 161 (1967) 556.

[11] LloYd, P., Proc. R. Soc. (London) 90 (1967) 207, 90 (1967) 217.

[12] GyoRfFy, B. L. and StotT, M. J. « Band Structure Spectroscopy of Metals and Alloys 》 (Ed. D. J. Fabian and L. M. Watson) 1973 p. 385.

[13] GyorfFy, B. L. and Stocks, G. M. (to be published).

[14] Roman, P. «Advanced Quantum Theory» (1965).

[15] Bansil, A., Ehrenreich, H., Schwartz, L. and Watson, R. (to be published).

[16] Korringa, J., J. Phys. \& Chem. Solids, 1 (1958) 252; Beeby, J. L., Proc. R. Soc. (London) A 279 (1964) 82, Phys. Rev. A 135, 130.

Soven, P., Phys. Rev. 151 (1966) 539;

SChwartz, L. M., Brouers, F., Vedyayev, A. V. and Ehrenreich, H., Phys. Rev. B 4 (1971) 3383.

[17] Arlingmauss, F. J., Phys. Rev. 157 (1967) 491.

[18] Stocks, G. M., Williams, R. W. and Faulkner, J. S., Phys. Rev. B 4 (1971) 2412. 